\title{
Modern role for clopidogrel in management of atrial fibrillation and stroke reduction
}

\author{
This article was published in the following Dove Press journal: \\ Vascular Health and Risk Management \\ 25 February 2010 \\ Number of times this article has been viewed
}

\section{Lars Maegdefessel \\ Junya Azuma \\ Philip S Tsao}

Department of Cardiovascular Medicine, School of Medicine, Stanford University, CA, USA
Correspondence: Lars Maegdefessel Division of Cardiovascular Medicine, Stanford University School of Medicine, 300 Pasteur Drive, Stanford,

CA 94305-5406, USA

Tel +l 6507245075

Fax +I 650725 I599

Email maegdefessel@stanford.edu
Abstract: Atrial fibrillation (AF) is the most common sustained cardiac arrhythmia. The prevalence of AF increases sharply in old age (prevalence approximately $10 \%$ among persons 80 years of age and older). The expected risk for ischemic stroke is increased five-fold by the presence of AF, primarily as a result of cardiogenic embolism. Multiple large-scale, randomized trials have been completed or are still underway to find optimal, efficacious, and relatively safe ways to reduce the risk of ischemic stroke and other systemic thromboembolic events related to AF. Antithrombotic strategies are accompanied by serious bleeding complications that threaten patients in need of medical stroke prevention. Treatment regimens for preventing thromboembolism in AF patients range from vitamin $\mathrm{K}$ antagonists such as warfarin or coumadins, antiplatelet drugs like aspirin or clopidogrel, to newly developed orally available antithrombotics like the direct thrombin inhibitor dabigatran, or the Factor Xa-inhibitor rivaroxaban. The available anticoagulant and antiplatelet drugs have different advantages and disadvantages. This review attempts to delineate the specific role of clopidogrel in patients with $\mathrm{AF}$ and at risk of stroke, taking into consideration new and ongoing trials in this important field of medical practice.

Keywords: clopidogrel, antiplatelet agents, atrial fibrillation, ischemic stroke

\section{Introduction}

Atrial fibrillation (AF) is the most common cardiac rhythm disorder and an important independent risk factor for ischemic stroke. AF affects nearly 2.5 million people in the US (approximately 1\%). The prevalence of this arrhythmia increases sharply with older age. Worldwide population trends of increasing life expectancy and increasing prevalence of known risk factors for $\mathrm{AF}$ indicate a worsening epidemic of the condition. ${ }^{1} \mathrm{AF}$ is uncommon among individuals $\leq 50$ years of age. In the Framingham Heart Study, the percentage risk of stroke ascribable to AF rose from $1.5 \%$ in the age group 50 to 59 years, to $23.5 \%$ in the age group 80 to 89 years. The median age of patients with AF is 72 years. Overall, AF accounts for about $15 \%$ of all strokes in the US. ${ }^{2}$ The rate of ischemic stroke among patients with AF included in primary prevention clinical trials and not treated with antithrombotic therapy averaged $4.5 \%$ per year, similar to estimates of stroke risk from the Framingham Heart Study. Further analyses from these studies indicate that the lifetime risk of AF for an individual aged 40 years is about $25 \%{ }^{3}$ The occurrence of AF may be even higher given the potential for $\mathrm{AF}$ to remain undiagnosed.

$\mathrm{AF}$ is more prevalent in men than in women at all ages. AF raises the risk of ischemic stroke four- to five-old, predominantly as the result of cardiogenic embolism. ${ }^{4}$ This is based on clinical assessment, by extension of operative findings of intracardiac

submit your manuscript $\mid$ www.dovepress.coin 
thrombus in patients with rheumatic mitral valve disease and, more recently, by transesophageal echocardiographic imaging of thrombus in the left atrium of patients with AF, mainly in the left atrial appendage. ${ }^{5}$

Given the epidemiologic characteristics of AF and the importance of stroke as a leading cause of death and disability, multiple large-scale, randomized trials have been completed, or are underway, to find effective and relatively safe ways to reduce the risk of ischemic stroke and other systemic thromboembolic events related to AF. Antagonists of vitamin $\mathrm{K}$ have been used as anticoagulants for over 50 years. Warfarin, a synthetic derivative of coumarin, is the most commonly used vitamin $\mathrm{K}$ antagonist (VKA) in the US. In most European countries, other coumarin derivatives (phenprocoumon and acenocoumarol) are used as an alternative to warfarin. Vitamin $\mathrm{K}$ is essential for the hepatic synthesis of Factors II (prothrombin), VII, IX, and X, as well as protein C and protein $\mathrm{S} .{ }^{6}$ With appropriate dosing, these medications effectively inhibit coagulation and have been shown to reduce substantially the risk of stroke in AF and the likelihood of recurrence of venous thromboembolism (VTE). ${ }^{7,8}$ However, VKAs are limited by a narrow therapeutic window as well as the need for frequent coagulation monitoring and dosage adjustments. Other drawbacks of this class include drug and food interactions (eg, foods rich in vitamin $\mathrm{K}$ ), a well documented incidence of major bleeding of $1 \%$ to $3 \%$, and delayed onset and offset of anticoagulant effect. ${ }^{9}$ However, in spite of their considerable limitations, VKAs have been the standard of care for long term prophylaxis of stroke in patients with AF.

Newly acquired data on alternative antithrombotic strategies might change this. The recently presented RE-LY trial, which compared the direct thrombin inhibitor dabigatran with warfarin, has the potential to set a new landmark in the prevention of stroke, thromboembolic complications, and reduction of life-threatening hemorrhagic complications. In patients with AF, dabigatran given at a dose of $110 \mathrm{mg}$ twice daily was associated with rates of stroke and systemic embolism that were similar to those associated with warfarin, as well as lower rates of major hemorrhage. Dabigatran administered at a dose of $150 \mathrm{mg}$ twice daily, as compared with warfarin, was associated with lower rates of stroke and systemic embolism but similar rates of major hemorrhage. ${ }^{10}$

Antiplatelet drugs such as acetylsalicylic acid (ASA), ticlopidine, dipyridamole, and clopidogrel play an important role in stroke prevention for patients with AF for various reasons and in specific indications. The purpose of this review is to elucidate the role of one of these antiplatelet drugs, clopidogrel, in stroke prevention for patients suffering from AF. We also focus on alternative antiaggregation therapies and their advantages and disadvantages.

\section{Pharmacology, pharmacokinetics, and mode of action}

Platelets are important in the initiation and progression of thrombus formation leading to thromboembolic obstruction of brain-supplying arterial blood vessels in patients with transient ischemic attack (TIA) and ischemic stroke. Antiplatelet agents have been confirmed in randomized trials as a cornerstone for medical stroke prevention in patients with TIA/stroke of non-cardioembolic etiology.

Clopidogrel is a prodrug administered orally. It is rapidly absorbed from the gastrointestinal tract with a bioavailability of about $50 \%{ }^{11}$ It is $98 \%$ bound to plasma proteins. The half-life of its active metabolite is approximately eight hours. Approximately $85 \%$ of the prodrug is hydrolyzed by esterases in the blood to an inactive carboxylic acid derivative, and only $15 \%$ of the prodrug is metabolized by the cytochrome P450 (CYP450) system in the liver to generate an active metabolite. ${ }^{12}$ The effect of liver disease on the antiplatelet activity of clopidogrel has not been thoroughly investigated. The active metabolite, a carboxylic acid derivative, irreversibly inhibits the adenosine diphosphate P2Y12 receptor. Activation of the $\mathrm{P} 2 \mathrm{X} 1$ and $\mathrm{P} 2 \mathrm{Y} 1$ receptors leads to alteration in shape and initiates a weak and transient phase of platelet aggregation. The P2X1 mediates extracellular calcium influx and utilizes adenosine triphosphate as an agonist (see Figure 1). Thienopyridines (such as clopidogrel or ticlopidine) bind highly selectively to the P2Y12-receptor on the surface of platelets. This binding is irreversible and therefore platelets exposed to thienopyridines are inhibited for their life spans. ${ }^{13}$ Therefore it takes 5-7 days to restore platelet function completely. This must be considered if clopidogrel is used in patients in whom invasive or surgical treatment is planned.

Given that ticlopidine has been associated with a high incidence of adverse events, clopidogrel has become the drug of choice. ${ }^{14}$ After repeated daily dosing with clopidogrel $75 \mathrm{mg}$, steady-state is achieved after 4-7 days. If a more rapid antiplatelet effect is required, a loading dose of $300 \mathrm{mg}$ clopidogrel followed by a daily dose of $75 \mathrm{mg}$ is needed. The efficacy of higher loading doses such as $600 \mathrm{mg}$ or $900 \mathrm{mg}$ need to be further investigated. At steady-state, clopidogrel inhibits $50 \%$ to $60 \%$ of platelet aggregation induced by adenosine diphosphate. ${ }^{15}$ 


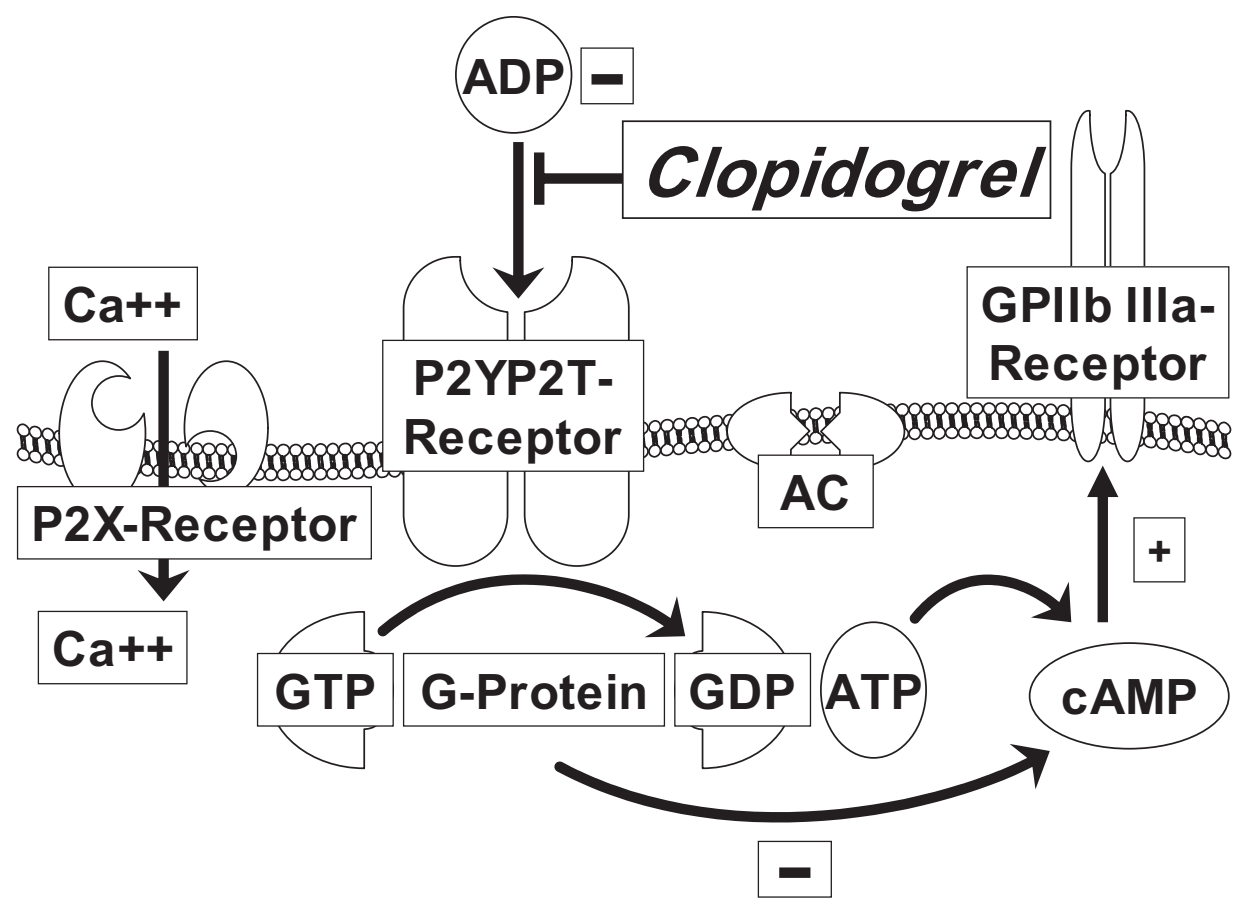

Figure I Mode of action of clopidogrel.

Clopidogrel irreversibly inhibits the ADP P2Y ${ }_{12}$ receptor. P2X mediates extracellular calcium influx and utilizes ATP as an agonist. The binding of ADP to the G-coupled $P 2 Y_{12}$ receptor liberates $\mathrm{G}$ protein subunits and results in stabilization of platelet aggregation. One subunit leads to inhibition of $A C$, which reduces cAMP levels. cAMP as well as the second subunit of the G protein lead to activation of the GP IIb/IIla receptor.

Abbreviations: AC, adenylyl cyclase; ADP, adenosine diphosphate; ATP, adenosine triphosphate; Ca, calcium; cAMP, cyclic adenosine monophosphate; GDP, guanosine diphosphate; GP, glycoprotein; GTP, guanosine triphosphate.

\section{Efficacy of clopidogrel for stroke reduction}

Platelet aggregation inhibitors reduce the risk of non-fatal stroke by $23 \%$ (from $10.8 \%$ to $8.3 \%$ over 36 months) and all vascular events (nonfatal myocardial infarction, nonfatal stroke, and vascular death) by $17 \%$ (from $21.4 \%$ to $17.8 \%$ over 29 months) in patients after TIA or stroke. ${ }^{16}$ Antiplatelet agents have different targets to prevent platelet activation and adhesion to the blood vessel wall or platelet aggregation finally resulting in thrombus formation. To date, ASA, the combination of aspirin (as the most common ASA) and extended-release dipyridamole and clopidogrel are most frequently used for long-term antiplatelet therapy in patients with non-cardioembolic stroke. ${ }^{17}$ Only aspirin has been shown to be safe and effective, both in the acute postischemic phase (first 48 hours; $160 \mathrm{mg}-300 \mathrm{mg} /$ day) and in long-term secondary prevention. ${ }^{18}$ As a consequence, where possible, aspirin should be started immediately in patients with TIA or ischemic stroke after brain hemorrhage has been ruled out by brain imaging. Aspirin is effective irrespective of dose (30 mg-300 mg/day) in long-term secondary stroke prevention, but doses $>150 \mathrm{mg} /$ day are associated with more side effects, especially gastrointestinal adverse events and bleeding. ${ }^{16}$
It is a common clinical practice to combine different antiplatelet agents to enhance their antiaggregation effect. The combination of aspirin 30-300 mg/day and extended-release dipyridamole $200 \mathrm{mg}$ twice a day was shown to be more effective than aspirin alone in the ESPS- 2 trial. ${ }^{19}$ A meta-analysis of five stroke prevention studies of aspirin alone versus aspirindipyridamole in 7612 patients with TIA or minor stroke showed a relative risk reduction in favor of the combination therapy for a combined vascular endpoint (ischemic stroke, myocardial infarction, vascular death) of $18 \%$ (95\% confidence interval [CI]: $8 \%-28 \%) .{ }^{20}$ The combination therapy was also more effective in preventing recurrent stroke alone. The clinically relevant side effect of headache in patients treated with dipyridamole can be reduced by slow titration and administration of dipyridamole. In the Prevention Regimen for Effectively Avoiding Second Strokes (PRoFESS) trial, ${ }^{21}$ the rate of permanent study discontinuation because of headache was $5.9 \%$ in the treatment arm receiving an aspirin-dipyridamole combination.

Clopidogrel $75 \mathrm{mg} /$ day was compared with aspirin $325 \mathrm{mg} /$ day in 19,185 patients with stroke, myocardial infarction, or peripheral arterial disease (PAD) in the Clopidogrel versus Aspirin in Patients at Risk of Ischemic Events (CAPRIE) study. ${ }^{22}$ During a mean followup period of 1.91 years, patients treated with clopidogrel had an annual 
$5.32 \%$ risk of ischemic stroke, myocardial infarction, or vascular death, compared with $5.83 \%$ in patients taking aspirin, resulting in a relative risk reduction of $8.7 \%(95 \%$ CI: $0.3-16.5)$. The greatest benefit of clopidogrel could be seen in patients with concomitant PAD.

Specific recommendations for antiplatelet therapy with clopidogrel can be made for patients who are allergic to aspirin and in patients at risk for stroke with PAD. In patients with a history of aspirin-induced ulcer bleeding, aspirin in combination with the proton-pump inhibitor (PPI) esomeprazole was found to be superior to clopidogrel alone in the prevention of recurrent ulcer bleeding. ${ }^{23}$ The combination of PPIs with clopidogrel resulted in a significantly increased risk of death and rehospitalization for acute coronary syndrome in patients after an acute myocardial infarction, as shown in a recently published retrospective study in 8205 patients. $^{24}$

A head-to-head comparison of clopidogrel versus the combination of aspirin $50 \mathrm{mg} /$ day and extended-release dipyridamole $400 \mathrm{mg}$ /day was made in the above-mentioned PRoFESS trial. ${ }^{21}$ This trial was the largest ever performed study of secondary stroke prevention, including 20,332 patients with recent ischemic stroke. There was no significant difference in the rate of recurrent stroke ( $8.8 \%$ versus $9.0 \%)$, or in efficacy across all other secondary endpoints or subgroup of patients after a mean followup period of 2.4 years. The aspirin-dipyridamole combination resulted in slightly more intracranial bleeds and a higher dropout rate because of headache compared with clopidogrel (5.9\% versus $0.9 \%)$.

The combination of aspirin-clopidogrel is not recommended for long-term secondary stroke prevention because of an increased bleeding risk. The Management of Atherothrombosis with Clopidogrel in High-risk Patients with Recent TIA or Ischemic Stroke (MATCH) trial compared the combination of clopidogrel $75 \mathrm{mg} /$ day and aspirin $75 \mathrm{mg} /$ day with clopidogrel monotherapy in 7599 high-risk patients with recent TIA/stroke, and failed to show superiority of combination therapy for the combined endpoint of stroke, myocardial infarction, vascular death, and hospitalization because of a vascular event. ${ }^{25}$ The combination resulted in a significant increase in life-threatening or major hemorrhage.

Similar to the results of the MATCH trial, the combined primary and secondary prevention Clopidogrel for High Atherothrombotic Risk and Ischemic Stabilization, Management, and Avoidance (CHARISMA) trial did not show a benefit for the combination of aspirin-clopidogrel over aspirin monotherapy. Again, bleeding rates were increased by the combination therapy. ${ }^{26}$ However, the subgroup of symptomatic patients with prior myocardial infarction, ischemic stroke, or symptomatic PAD showed a benefit from combination antiplatelet therapy, ${ }^{27}$ but this finding has to be confirmed in a prospective, randomized trial.

The Atrial Fibrillation Clopidogrel Trial with Irbesartan for Prevention of Vascular Events (ACTIVE) compared the addition of either clopidogrel $75 \mathrm{mg}$ /day or placebo to aspirin $75 \mathrm{mg}-100 \mathrm{mg} /$ day for use in patients with AF who were considered "unsuitable" for VKA therapy. ${ }^{28}$ ACTIVE A was one of three related studies of patients with AF, including ACTIVE $\mathrm{W},{ }^{29}$ a randomized comparison of a VKA versus a combination of clopidogrel- aspirin, and ACTIVE I, an ongoing, placebo-controlled study of irbesartan for patients enrolled in ACTIVE A or ACTIVE W. ACTIVE W was terminated early after showing that VKA therapy was more effective than the clopidogrel-aspirin combination in patients at high risk for stroke, confirming the superiority of this strategy.

ACTIVE A added to our understanding of the role of intensive antiplatelet therapy to prevent stroke in selected patients with AF. This large, randomized trial showed that the combination of clopidogrel-aspirin significantly reduced the rate of major vascular events (driven primarily by fewer strokes) compared with aspirin alone, in certain patients with AF. One disabling or fatal stroke would be prevented per approximately 200 patients treated for one year with a clopidogrel-aspirin combination. These effects were mostly consistent across subgroups, although there may be interactions with age, $\mathrm{CHADS}_{2}$ score (see Table), or previous use of a VKA. On the other hand, one extra major bleeding episode and one extra intracranial hemorrhage would occur per approximately 143 and 500 patients, respectively, treated for one year with a clopidogrel-aspirin combination.

The issue of to whom do the ACTIVE A results best apply cannot be completely answered at this point. Although the investigators intended to enroll patients who were ineligible for anticoagulation therapy with VKAs, less than one quarter of participants had a documented contraindication to VKAs. Half were enrolled on the basis of a physician's judgment that a VKA was not suitable for the patient; however, no strict case definition for "unsuitable" was used. Patient preference not to take a VKA was the reason given for enrollment of the approximately $25 \%$ remaining participants, but there is evidence suggesting that this preference can be affected by how therapeutic options are presented to patients.

It is important to note that neither regimen studied in ACTIVE A is as effective as VKA therapy for the prevention 


\section{Table I CHADS ${ }_{2}$ score}

\begin{tabular}{|c|c|c|c|}
\hline $\mathrm{C}:$ & \multicolumn{2}{|c|}{ Congestive heart failure } & = I point \\
\hline $\mathrm{H}:$ & \multicolumn{2}{|c|}{ Hypertension (systolic > $160 \mathrm{mmHg}$ ) } & $=\mathrm{I}$ point \\
\hline A: & \multicolumn{2}{|c|}{ Age $>75$ years } & $=\mathrm{I}$ point \\
\hline D: & \multicolumn{2}{|c|}{ Diabetes } & $=\mathrm{I}$ point \\
\hline S: & \multicolumn{2}{|c|}{ Prior transient ischemic attack or stroke } & $=2$ points \\
\hline \multicolumn{2}{|c|}{ CHADS $_{2}$ score } & \multicolumn{2}{|c|}{ Adjusted stroke rate* $(\%)[95 \% \mathrm{Cl}]$} \\
\hline 0 & \multicolumn{3}{|c|}{$1.9(1.2-3.0)$} \\
\hline I & \multicolumn{3}{|c|}{$2.8(2.0-3.8)$} \\
\hline 2 & \multicolumn{3}{|c|}{$4.0(3.1-5.1)$} \\
\hline 3 & \multicolumn{3}{|c|}{$5.9(4.6-7.3)$} \\
\hline 4 & \multicolumn{3}{|c|}{$8.5(6.3-11.1)$} \\
\hline 5 & \multicolumn{3}{|c|}{$12.5(8.2-17.5)$} \\
\hline 6 & \multicolumn{3}{|c|}{$18.2(10.5-27.4)$} \\
\hline
\end{tabular}

Notes: The annual stroke rates for patients suffering from atrial fibrillation according to the $\mathrm{CHADS}_{2}$ Score. Patients with a CHADS, Score of 0 are considered to have a low risk for cardioembolic stroke. Patients with a score of $\mathrm{I}-2$ are classified as having a moderate risk for stroke and should be medically treated with an antithrombotic agent. Patients with a score of 3 or higher have a high risk for thromboembolic complications and require optimal antithrombotic treatment as well. $*$ The adjusted stroke rate is the expected stroke rate per 100 person/years derived from the multivariable model assuming that aspirin is not taken.

of ischemic stroke. The annual rates of stroke among participants receiving the clopidogrel-aspirin combination $(2.4 \%)$ or aspirin alone $(3.3 \%)$ were notably higher than those reported in patients at high risk for stroke who received VKA therapy (approximately 1.1\%-1.3\%). These high stroke rates were seen despite the fact that nearly $40 \%$ of ACTIVE A participants had a $\mathrm{CHADS}_{2}$ score of 0 or 1 (low predicted risk of stroke), and $34 \%$ had a $\mathrm{CHADS}_{2}$ score of 2 (moderate predicted risk of stroke). Although aspirin therapy, with or without clopidogrel, requires no dose adjustment or monitoring (two of the main drawbacks of VKAs), a surprisingly high percentage of patients discontinued therapy during the followup period (39\% in the clopidogrel group and 37\% in the placebo group). Adherence is usually worse in everyday clinical practice than in selected patients in carefully monitored trial protocols, so the benefits seen with clopidogrel in ACTIVE A may not be achieved in "real-world" clinical populations.

\section{Safety and tolerability}

Several complications are common with all antiplatelet agents. Severe rash and diarrhea are more frequently associated with clopidogrel than with aspirin. The incidence of hemorrhagic events is comparable between aspirin and clopidogrel. Gastrointestinal bleeding occurs with both agents. However, substitution of clopidogrel for aspirin in high-risk patients with recurrent ulcer bleeding is not recommended because it is inferior to treatment with aspirin and a PPI. ${ }^{23}$ A serious but less frequent adverse reaction of clopidogrel is intracranial hemorrhage. Furthermore, clopidogrel is associated with thrombotic thrombocytopenic purpura. ${ }^{30}$ Rates of neutropenia and thrombotic thrombocytopenic purpura are similar between aspirin and clopidogrel. Thrombotic thrombocytopenic purpura occurs within the first two weeks of treatment. Ticlopidine, another thienopyridine, is associated with neutropenia in approximately $1 \%$ of patients, which is mostly reversible on discontinuation of therapy. ${ }^{31}$ However, in a few cases it is irreversible and potentially life-threatening. Patients must therefore be regularly monitored within the first three months of treatment. Clopidogrel is contraindicated in patients hypersensitive to ticlopidine, and in those with active gastrointestinal bleeding, acute hemorrhagic stroke or severe hepatic impairment. ${ }^{32}$

Clopidogrel is associated with an increased risk of serious bleeding complications. This potentially becomes a problem with patients undergoing percutaneous coronary intervention or bypass surgery. The risk of reoperation and requirement for blood transfusions during coronary artery bypass surgery is increased. ${ }^{33}$ The concurrent use of clopidogrel with other platelet inhibitors, anticoagulants, fibrinolytics, or non-steroidal anti-inflammatory agents is associated with an increased incidence of bleeding. To reduce the risk for gastrointestinal bleeding, guidelines recommend the addon use of a PPI. ${ }^{34}$ However, a recent trial has shown that certain PPIs (namely omeprazole, lansoprazole, and rabeprazole) interfere with the activating metabolism of clopidrogrel. ${ }^{35}$ This results in decreased antiplatelet activity of clopidogrel, and patients treated with one of these PPIs were at a significant higher risk of readmission for MI.

The optimal antithrombotic treatment for patients with AF at medium or high thromboembolic risk who undergo percutaneous coronary intervention with stent implantation is currently undefined. Based on the scant available data, triple therapy consisting of VKA-aspirin-clopidogrel appears to offer the best protection against thromboembolic and myocardial ischemic events, at the price of an increased risk of serious hemorrhagic complications. ${ }^{36}$ The VKA-aspirin combination appears to be less effective, and therefore should not be prescribed in the early period following percutaneous coronary intervention with stent implantation. ${ }^{37}$ Whether the VKA-clopidogrel combination will preserve efficacy with less bleeding is an important and challenging question, but initial data support its use for several months after the early period of triple therapy for the prevention of recurrent coronary events. ${ }^{38}$ 
The safe use of oral anticoagulation with VKAs depends on patient cooperation and a monitoring system that can achieve INR targets on a regular basis. Findings of the randomized trials suggest that anticoagulation at an INR of 2.0-3.0 can be reasonably safe even for elderly patients, and the Italian Study on Complications of Oral Anticoagulant Therapy (ISCOAT) study ${ }^{39}$ and Anticoagulation and Risk Factors in Atrial Fibrillation (ATRIA) study ${ }^{40}$ studies demonstrate that low hemorrhage rates can be achieved in clinical practice outside of trials, particularly if well organized health professionals are involved.

\section{Patient considerations}

The $\mathrm{CHADS}_{2}$ score is the best validated clinical predictor of risk of stroke and of who should be treated with antithrombotics. ${ }^{41}$ It assigns points ( $(0-6)$ depending on the presence or absence of comorbidities (see Table 1). To compensate for the increased risk of stroke, anticoagulation may be necessary. However, with VKAs, if a patient with AF has a yearly risk of stroke of less than $2 \%$, the risks associated with taking warfarin outweigh the risk of having a stroke. For patients with a lower risk for ischemic stroke, antiplatelet agents such as aspirin or clopidogrel can be prescribed. ${ }^{42}$

In addition to clinical risk stratification, patient perspectives and preferences should be incorporated into the decision about the most suitable antithrombotic therapy. Prior studies have shown that patient and physician perspectives often differ, with patients, unlike physicians generally placing more value on stroke prevention than avoiding a major hemorrhage. Many patients, in fact, assign values to a moderate to severe stroke that are equivalent to or worse than death. ${ }^{16}$ Ethnic and cultural differences in patient perception of AF and antithrombotic therapy are known to exist, and these differences can affect worldwide use of antithrombotic therapy in AF patients. ${ }^{43}$

Decision-analysis techniques have been used to evaluate the projected net benefit or harm associated with different antithrombotic treatment strategies in AF. These models formally combine the absolute risks associated with a number of patient characteristics, estimates of the efficacy and safety of antithrombotic treatment, and assigned values (utilities) of related health states (eg, taking warfarin, suffering a major stroke) trials. Sensitivity analyses test the impact of varying assumptions made in the model. In general, published decision analyses support the net benefit of anticoagulation with oral VKAs for patients with $\mathrm{AF}$ at moderate to high risk for stroke but not at very high risk of bleeding. ${ }^{44}$ However, the treatment threshold for these levels of risk and the criteria for moderate and high-risk categories vary across studies, reflecting the need for more refined estimates. The decision-analysis approach has been modified in attempts to help individual patients make better choices about antithrombotic therapy in AF. ${ }^{16}$ Strong evidence is currently lacking, however, that these decision support tools improve clinical outcomes.

Multiple studies of practice patterns of use of antithrombotic and antiplatelet therapy for AF have been reported. In North America and Western Europe the use of VKAs for AF has increased greatly from the early 1990s to the present. Currently, at least $50 \%$ of $\mathrm{AF}$ patients are treated with VKAs, and their use is moderately higher in patients at increased risk for ischemic stroke and moderately lower in patients at increased risk of bleeding. However, the use of VKAs decreases with age $\geq 80$ years despite the fact that such patients are at higher risk of ischemic stroke. Many patients at apparently low risk for stroke are treated with VKAs and many patients at higher risk for stroke, eg, those who have a past history of ischemic stroke, are not treated with VKAs. Detailed clinical assessment of highrisk patients not receiving VKA therapy shows that many of them have clear physical and/or cognitive contraindications to anticoagulants. ${ }^{45}$ In contrast with the generally aggressive use of anticoagulants for AF in North America and Western Europe, physicians in Japan are reluctant to prescribe VKAs for AF, presumably reflecting more concern about hemorrhagic stroke. When VKAs are prescribed in Japan, target INR levels are generally lower. ${ }^{46}$

For a long time oral VKAs were the most effective form of stroke prevention therapy in patients with AF. However, VKA therapy approximately doubles the risk of intracranial haemorrhage, is challenging to deliver in a high-quality fashion (ie, maintaining an international normalized INR ratio of 2.0 to 3.0), and can diminish quality of life because of the need for frequent testing, dietary restrictions, and drug-drug interactions. ${ }^{47}$ The situation is exacerbated by difficulties in predicting which patients are at highest risk for stroke and who are at highest risk for bleeding. As previously mentioned, preferences of physicians and patients may differ substantially with regard to the relative importance of avoiding ischemic stroke and avoiding bleeding complications. Collectively, these factors have contributed to marked variation in the use of different antithrombotic agents for patients with $\mathrm{AF}$ and have fueled the pursuit of alternative stroke prevention strategies.

As a result, patient education and involvement in the anticoagulation decision is important. Many patients with AF have a fear of ischemic stroke and choose a VKA despite the relatively small decrease in the absolute risk of stroke, while 
others at relatively low risk of stroke are likely to want to avoid the burden and risks of VKA therapy and so opt instead for antiplatelet agents, such as clopidogrel or aspirin.

\section{Conclusions}

Fifteen years after the publication of multiple definitive trials demonstrating the efficacy of anticoagulants in preventing stroke in patients with $\mathrm{AF}$ and more recent evidence from large and rigorous trials which have validated both the efficacy and safety of anticoagulants in AF, there remains considerable controversy about which patients with AF should be treated with long-term VKA and those who should receive antiplatelet therapy.

Indeed, some recent revised guidelines have become more restrictive in recommending anticoagulant therapy for patients with AF. However, ASAs (such as aspirin) are the most widely used antiplatelet agents in patients with $\mathrm{AF}$ who are at risk for stroke. For patients who develop TIA/stroke on ASA therapy, the options are to increase the ASA dosage, to add another antiplatelet agent, to switch to another antiplatelet agent, or to switch to another antiaggregation agent such as a VKA or direct thrombin inhibitor (dabigatran being the only approved agent for this indication to date). One proposed approach is to combine antiplatelet agents with different modes of action, such as ASA and clopidogrel, to achieve a better antithrombotic effect. Several trials, including PRoFESS, MATCH and CHARISMA $^{21,25,26}$ addressed this issue. None of these trials demonstrated a clear benefit of a combination therapy of antiplatelet agents over monotherapy. Furthermore, bleeding rates were increased with all combination therapies. In patients with $\mathrm{AF}$ and a lower risk for stroke who are intolerant of ASAs, clopidogrel can be a useful alternative, especially when the risk of hemorrhage is potentially increased by VKA therapy.

Approximately a third of patients with $\mathrm{AF}$ also have a diagnosis of coronary artery disease. ${ }^{16}$ These patients face a sizable risk of future coronary events as well as stroke. For those individuals who are receiving anticoagulants to prevent stroke, it is unclear if an ASA and/or clopidogrel should be added to prevent coronary events and stroke more effectively. There are no randomized trials that directly address this issue. Decisions on optimal antithrombotic and antiplatelet therapy need to be made individually, taking into consideration the severity of coronary artery disease, stent implantation, and risk of stroke.

To further optimize stroke prevention in patients with $\mathrm{AF}$, more reliable methods to predict the individual risk of a patient having an ischemic stroke and bleeding (especially intracranial hemorrhage) need to be identified. Physicians should consistently apply existing data to identify patients eligible for either VKA or antiplatelet therapy, and should present a complete and balanced picture of the absolute risks and benefits of each approach to the patient to enable informed decision-making. To date, current evidence supports the view that patients with $\mathrm{AF}$ at moderate-to-high risk (estimated favorably with the $\mathrm{CHADS}_{2}$ score) for stroke, and for whom a VKA is suitable, should be considered for this therapy to maximize the prevention of thromboembolism with an acceptable risk of major bleeding. For patients with $\mathrm{AF}$ who are at moderate-to-high risk for stroke but who are not suitable for VKA therapy, and in whom high-quality anticoagulation is not achieved despite the best efforts, or those who are at lower risk for stroke, the combination of clopidogrel-aspirin will most likely provide more clinical benefit than aspirin alone.

As mentioned earlier, the RE-LY trial ${ }^{10}$ has the potential to change the landscape of stroke prevention in patients with AF. The advantages of treatment with an orally available direct thrombin inhibitor such as dabigatran are obvious. Dabigatran more closely meets the criteria for optimal antithrombotic efficacy and has the ability to improve quality of care. Such an advance might also help to overcome the gaps between evidence-based treatment recommendations and clinical practice. Dabigatran has proven to be highly effective in reducing venous thromboembolism, ${ }^{48}$ inhibiting both free and clot-bound coagulation factors. Furthermore, it has a predictable dose response and kinetics, and shows low, non-specific plasma protein binding, resulting in a low rate of bleeding events. Coagulation monitoring and dose adjustment is not routinely required, thus creating a wide therapeutic window. Furthermore, there is little interaction with food or other medicines. ${ }^{49}$ Overall, newly developed and orally available direct thrombin inhibitors and/or Factor $\mathrm{Xa}$ inhibitors may reduce the role of antiplatelet agents such as clopidogrel, as well as VKAs such as warfarin, and overcome obstacles of current antithrombotic strategies in stroke prevention for patients suffering from AF.

\section{Disclosures}

The authors declare no competing interests in this work.

\section{References}

1. Go AS, Hylek EM, Phillips KA, et al. Prevalence of diagnosed atrial fibrillation in adults: National implications for rhythm management and stroke prevention: the AnTicoagulation and Risk Factors in Atrial Fibrillation (ATRIA) Study. JAMA. 2001;285:2370-2375. 
2. Schnabel RB, Sullivan LM, Levy D, et al. Development of a risk score for atrial fibrillation (Framingham Heart Study): A community-based cohort study. Lancet. 2009;373:739-745.

3. Hart RG, Pearce LA, Aguilar MI. Meta-analysis: Antithrombotic therapy to prevent stroke in patients who have nonvalvular atrial fibrillation. Ann Intern Med. 2007;146:857-867.

4. Lip G, Nieuwlaat R, Pisters R, Lane D, Crijns H. Refining clinical risk stratification for predicting stroke and thromboembolism in atrial fibrillation using a novel risk factor based approach: The Euro Heart Survey on Atrial Fibrillation. Chest. 2010;137;263-272.

5. Nieuwlaat R, Olsson SB, Lip GY, et al; Euro Heart Survey Investigators. Guideline-adherent antithrombotic treatment is associated with improved outcomes compared with undertreatment in high-risk patients with atrial fibrillation. The Euro Heart Survey on Atrial Fibrillation. Am Heart J. 2007;153:1006-1012.

6. Hirsh J, Dalen J, Anderson DR, et al. Oral anticoagulants: Mechanism of action, clinical effectiveness, and optimal therapeutic range. Chest. 2001;119(Suppl):S8-S21.

7. Haas S. New oral Xa and IIa inhibitors: Updates on clinical trial results. J Thromb Thrombolysis. 2008;25:52-60.

8. Bates SM, Ginsberg JS. Clinical practice. Treatment of deep vein thrombosis. N Engl J Med. 2004;351:268-277.

9. Mackman N. Triggers, targets and treatments for thrombosis. Nature. 2008;451:914-918.

10. Connolly SJ, Ezekowitz MD, Yusuf S, et al. RE-LY Steering Committee and Investigators. Dabigatran versus warfarin in patients with atrial fibrillation. N Engl J Med. 2009;361:1139-1151.

11. Patrono C, Baigent C, Hirsh J, Roth G; American College of Chest Physicians. Antiplatelet drugs: American College of Chest Physicians evidence-based clinical practice guidelines. 8th ed. Chest. 2008; 133(Suppl):199-233.

12. Wallentin L. P2Y(12) inhibitors: Differences in properties and mechanisms of action and potential consequences for clinical use. Eur Heart J. 2009;30:1964-1977.

13. Porto I, Giubilato S, De Maria GL, Biasucci LM, Crea F. Platelet P2Y12 receptor inhibition by thienopyridines: Status and future. Expert Opin Investig Drugs. 2009;18:1317-1332.

14. Hashemzadeh M, Goldsberry S, Furukawa M, Khoynezhad A, Movahed MR. ADP receptor-blocker thienopyridines: Chemical structures, mode of action and clinical use. A review. J Invasive Cardiol. 2009;21:406-412.

15. Motovska Z, Widimsky P. Clopidogrel before elective percutaneous coronary intervention. J Clin Pharmacol. 2009; Sep 29 [Epub ahead of print].

16. Singer DE, Albers GW, Dalen JE, et al. American College of Chest Physicians. Antithrombotic therapy in atrial fibrillation: American College of Chest Physicians evidence-based clinical practice guidelines. 8th ed. Chest. 2008;133(Suppl):546-592.

17. Levi M, Hobbs FD, Jacobson AK, et al. Improving antithrombotic management in patients with atrial fibrillation: Current status and perspectives. Semin Thromb Hemost. 2009;35:527-542.

18. Leys D, Balucani C, Cordonnier C. Antiplatelet drugs for ischemic stroke prevention. Cerebrovasc Dis. 2009;27:1:120-125.

19. Vane JR, Meade TW. Second European Stroke Prevention Study (ESPS 2): Clinical and pharmacological implications. J Neurol Sci. 1997;145:123-125.

20. Tijssen JG. Low-dose and high-dose acetylsalicylic acid, with and without dipyridamole: A review of clinical trial results. Neurology. 1998;51:S15-S16.

21. Diener HC, Sacco RL, Yusuf S, et al. Prevention Regimen for Effectively Avoiding Second Strokes (PRoFESS) study group. Effects of aspirin plus extended-release dipyridamole versus clopidogrel and telmisartan on disability and cognitive function after recurrent stroke in patients with ischaemic stroke in the Prevention Regimen for Effectively Avoiding Second Strokes (PRoFESS) trial: A double-blind, active and placebocontrolled study. Lancet Neurol. 2008;7:875-884.
22. Ferguson JJ 3rd, Gonzalez ER, Kannel WB, Olin JW, Raps EC. Clinical safety and efficacy of clopidogrel - implications of the Clopidogrel versus Aspirin in Patients at Risk of Ischemic Events (CAPRIE) study for future management of atherosclerotic disease. Clin Ther. 1998;20:42-53.

23. Lai KC, Chu KM, Hui WM, et al. Esomeprazole with aspirin versus clopidogrel for prevention of recurrent gastrointestinal ulcer complications. Clin Gastroenterol Hepatol. 2006;4:860-865.

24. Ho PM, Maddox TM, Wang L, et al. Risk of adverse outcomes associated with concomitant use of clopidogrel and proton pump inhibitors following acute coronary syndrome. JAMA. 2009;301:937-944.

25. Diener HC, Bogousslavsky J, Brass LM, et al; MATCH investigators. Aspirin and clopidogrel compared with clopidogrel alone after recent ischaemic stroke or transient ischaemic attack in high-risk patients (MATCH): Randomised, double-blind, placebo-controlled trial. Lancet. 2004;364:331-337.

26. Bhatt DL, Fox KA, Hacke W, et al; CHARISMA Investigators. Clopidogrel and aspirin versus aspirin alone for the prevention of atherothrombotic events. N Engl J Med. 2006;354:1706-1717.

27. Bhatt DL, Flather MD, Hacke W, et al; CHARISMA Investigators. Patients with prior myocardial infarction, stroke, or symptomatic peripheral arterial disease in the CHARISMA trial. J Am Coll Cardiol. 2007;49:1982-1988.

28. Connolly S, Yusuf S, Budaj A, et al. Rationale and design of ACTIVE: The atrial fibrillation clopidogrel trial with irbesartan for prevention of vascular events. Am Heart J. 2006;151:1187-1193.

29. Connolly S, Pogue J, Hart R, et al. Clopidogrel plus aspirin versus oral anticoagulation for atrial fibrillation in the Atrial fibrillation Clopidogrel Trial with Irbesartan for prevention of Vascular Events (ACTIVE W): A randomised controlled trial. Lancet. 2006;367:1903-1912.

30. Bennett CL, Connors JM, Carwile JM, et al. Thrombotic thrombocytopenic purpura associated with clopidogrel. $N$ Engl J Med. 2000;342:1773-1777.

31. Zakarija A, Kwaan HC, Moake JL, et al. Ticlopidine- and clopidogrelassociated thrombotic thrombocytopenic purpura (TTP): Review of clinical, laboratory, epidemiological, and pharmacovigilance findings (1989-2008). Kidney Int Suppl. 2009;112:S20-S24.

32. Bennett CL, Kim B, Zakarija A, et al; SERF-TTP Research Group. Two mechanistic pathways for thienopyridine-associated thrombotic thrombocytopenic purpura: A report from the SERF-TTP Research Group and the RADAR Project. J Am Coll Cardiol. 2007;50:1138-1143.

33. Carrel T, Englberger L. Coronary bypass surgery in patients treated with clopidogrel. Is it safe? Curr Opin Cardiol. Epub ahead of print 2009, Aug 13.

34. Anderson JL, Adams CD, Antman EM, et al. American College of Cardiology; American Heart Association Task Force on Practice Guidelines. ACC/AHA 2007 guidelines for the management of patients with unstable angina/non-ST-elevation myocardial infarction: A report of the American College of Cardiology/American Heart Association Task Force on Practice Guidelines. J Am Coll Cardiol. 2007;50:1-157.

35. Juurlink DN, Gomes T, Ko DT, et al. A population-based study of the drug interaction between proton pump inhibitors and clopidogrel. CMAJ. 2009;180:713-718.

36. Rubboli A, Halperin JL, Airaksinen J, et al. Antithrombotic therapy in patients treated with oral anticoagulation undergoing coronary artery stenting. An expert consensus document with focus on atrial fibrillation. Annals of Medicine. 2008;40:428-436.

37. Maegdefessel L, Schlitt A, Faerber J, et al. Anticoagulant and/or antiplatelet treatment in patients with atrial fibrillation after percutaneous coronary intervention. A single-center experience. Med Klin. 2008;103:628-632.

38. Karjalainen PP, Porela P, Ylitalo A, Vikman S, Nyman K, Vaittinen MA, et al. Safety and efficacy of combined antiplatelet-warfarin therapy after coronary stenting. Eur Heart J. 2007;28:726-732. 
39. Palareti G, Manotti C, DAngelo A, et al. Thrombotic events during oral anticoagulant treatment: Results of the inception-cohort, prospective, collaborative ISCOAT study. ISCOAT study group (Italian Study on Complications of Oral Anticoagulant Therapy). Thromb Haemost. 1997;78:1438-1443.

40. Go AS, Hylek EM, Borowsky LH, Phillips KA, Selby JV, Singer DE. Warfarin use among ambulatory patients with nonvalvular atrial fibrillation: The anticoagulation and risk factors in atrial fibrillation (ATRIA) study. Ann Intern Med. 1999;131:927-934.

41. Gage BF, Waterman AD, Shannon W, Boechler M, Rich MW, Radford MJ. Validation of clinical classification schemes for predicting stroke: Results from the National Registry of Atrial Fibrillation. JAMA 2001;285:2864-2870.

42. Gage BF, van Walraven C, Pearce L, et al. Selecting patients with atrial fibrillation for anticoagulation: Stroke risk stratification in patients taking aspirin. Circulation. 2004;110:2287-2292.
43. Pisters R, de Vos CB, Nieuwlaat R, Crijns HJ. Use and underuse of oral anticoagulation for stroke prevention in atrial fibrillation: Old and new paradigms. Semin Thromb Hemost. 2009;35:554-559.

44. Levi M, de Peuter OR, Kamphuisen PW. Management strategies for optimal control of anticoagulation in patients with atrial fibrillation. Semin Thromb Hemost. 2009;35:560-567.

45. Hylek EM. Antithrombotic prophylaxis in elderly patients with atrial fibrillation. Semin Thromb Hemost. 2009;35:548-553.

46. MacDougall NJ, Amarasinghe S, Muir KW. Secondary prevention of stroke. Expert Rev Cardiovasc Ther. 2009;7:1103-1115.

47. Bousser MG. Antithrombotic agents in the prevention of ischemic stroke. Cerebrovasc Dis. 2009;27:12-19.

48. Eikelboom JE, Weitz JI. Dabigatran etexilate for prevention of venous thromboembolism. Thromb Haemost. 2009;101:2-4.

49. Haas S. New anticoagulants - towards the development of an "ideal" anticoagulant. Vasa. 2009;38:13-29.
Vascular Health and Risk Management

\section{Publish your work in this journal}

Vascular Health and Risk Management is an international, peerreviewed journal of therapeutics and risk management, focusing on concise rapid reporting of clinical studies on the processes involved in the maintenance of vascular health; the monitoring, prevention and treatment of vascular disease and its sequelae; and the involvement of

\section{Dovepress}

metabolic disorders, particularly diabetes. This journal is indexed on PubMed Central and MedLine. The manuscript management system is completely online and includes a very quick and fair peer-review system, which is all easy to use. Visit http://www.dovepress.com/ testimonials.php to read real quotes from published authors

Submit your manuscript here: http://www.dovepress.com/vascular-health-and-risk-management-journal 